\title{
Growth differentiation factor-15 is a predictor of important disease outcomes in patients with COPD
}

\author{
Gunnar R. Husebø (1) ${ }^{1,2}$, Rune Grønseth", Lorena Lerner ${ }^{3}$, Jeno Gyuris ${ }^{3}$, \\ Jon A. Hardie ${ }^{2}$, Per S. Bakke ${ }^{2}$ and Tomas M. Eagan ${ }^{1,2}$
}

Affiliations: ${ }^{1}$ Dept of Thoracic Medicine, Haukeland University Hospital, Bergen, Norway. ${ }^{2}$ Dept of Clinical Science, University of Bergen, Bergen, Norway. ${ }^{3}$ AVEO Oncology, Cambridge, MA, USA.

Correspondence: Gunnar R. Husebø, Dept of Thoracic Medicine, Haukeland University Hospital, 5021 Bergen, Norway. E-mail: Gunnar.HuseboQuib.no

@ERSpublications

In COPD, GDF15 predicts a higher mortality, increased COPD exacerbation rate and a faster decline in FEV1 and FVC http://ow.ly/yDgn307n0SO

Cite this article as: Husebø GR, Grønseth R, Lerner L, et al. Growth differentiation factor-15 is a predictor of important disease outcomes in patients with COPD. Eur Respir J 2017; 49: 1601298 [https://doi.org/ $10.1183 / 13993003.01298-2016]$.

ABSTRACT Increased levels of growth differentiation factor-15 (GDF15) are associated with cachexia, cardiovascular disease and all-cause mortality. The role of GDF15 in chronic obstructive pulmonary disease (COPD) is unknown.

The study included 413 patients with COPD from the Bergen COPD Cohort Study. All patients had a forced expiratory volume in $1 \mathrm{~s}\left(\mathrm{FEV}_{1}\right)<80 \%$ predicted, a FEV1 to forced vital capacity (FVC) ratio $<0.7$ and a history of smoking. Spirometry, fat free mass index, blood gases and plasma GDF15 were measured at baseline. Patients were followed for 3 years regarding exacerbations and changes in lung function, and 9 years for mortality. Yearly exacerbation rate, survival and yearly change in FEV1/FVC were evaluated with regression models.

Median plasma GDF15 was $0.86 \mathrm{ng} \cdot \mathrm{mL}^{-1}$ (interquartile range $0.64-1.12 \mathrm{ng} \cdot \mathrm{mL}^{-1}$ ). The distribution was not normal and GDF15 was analysed as a categorical variable. High levels of GDF15 were associated with a higher exacerbation rate (incidence rate ratio $1.39,95 \%$ CI 1.1-1.74, $\mathrm{p}=0.006$, adjusted values). Furthermore, high levels of GDF15 were associated with higher mortality (hazard ratio 2.07, 95\% CI $1.4-3.1, \mathrm{p}<0.001)$ and an increased decline in both FEV1 $(4.29 \%$ versus $3.25 \%)$ and FVC $(2.63 \%$ versus $1.44 \%$ ) in comparison to low levels ( $\mathrm{p}<0.01$ for both).

In patients with COPD, high levels of GDF15 were independently associated with a higher yearly rate of exacerbations, higher mortality and increased decline in both FEV1 and FVC.

This article has supplementary material available from erj.ersjournals.com

Received: June 302016 | Accepted after revision: Dec 012016

Conflict of interest: Disclosures can be found alongside this article at erj.ersjournals.com 


\section{Introduction}

Chronic obstructive pulmonary disease (COPD) is among the world's leading causes of disability and death, and has a worldwide prevalence expected to increase for the next decade [1]. COPD is a heterogeneous disease, illustrated by the presence of different phenotypes [2], as well as large variation in morbidity, with patients with end-stage COPD often having frequent exacerbations, cachexia and a high mortality [3].

Growth differentiation factor-15 (GDF15) is a cytokine of the transforming growth factor $\beta$-family, also known as macrophage inhibitory cytokine 1 (MIC-1). GDF15 is a $25-\mathrm{kDa}$ protein initially discovered in macrophages but also found in epithelial tissues and plasma in a physiologic state [4-7].

In recent years, GDF15 has emerged as a biomarker for cardiovascular disease [8], diabetes [9], renal failure [10,11] and several different malignancies [12-14]. Elevated levels of GDF15 are associated with increased disease severity and poor prognosis [15], but the underlying pathophysiology is unknown. GDF15 is also a marker of all-cause mortality [16] and is believed to be a mediator of cachexia both in cancer and chronic disease [17-20].

As a cytokine associated with both inflammation and cachexia, along with increased mortality, GDF15 is of interest in patients with COPD, both as a diagnostic or prognostic biomarker and as a potential treatment target. A few smaller studies have examined GDF15 in COPD, indicating elevated plasma levels of GDF15 in patients with COPD [21, 22], and an additional increase during COPD exacerbations [21, 23, 24]. Nevertheless, the role of GDF15 as a prognostic biomarker in COPD is still unclear.

The aims of our study were to examine the relationship between systemic levels of GDF15 and important COPD characteristics such as lung function, exacerbations, cachexia and mortality, using both cross-sectional and longitudinal data from a large cohort of patients with COPD.

\section{Methods}

\section{Study population}

The Bergen COPD Cohort Study (BCCS) is a follow-up study from Western Norway started in 2006 that includes 433 patients with COPD and 325 subjects without COPD at baseline. All patients were examined at our study centre in Bergen, with attempts made to follow-up in regular intervals for 3 years. At all visits we took samples to contribute to the study biobank. The BCCS was designed foremost to examine predictors of COPD progression; details regarding study sampling and data collection have previously been described [25-27]. For the current study, we included the 413 patients with COPD who did not use oral steroids at the time of inclusion $(n=11)$ and for whom we had remaining plasma samples in our biobank. All patients were between 41 and 76 years at inclusion, and were former or current smokers. The patients had a clinical diagnosis of COPD, evaluated by a study physician, and a post-bronchodilation forced expiratory volume in $1 \mathrm{~s}(\mathrm{FEV} 1)$ to forced vital capacity (FVC) ratio $<0.7$ and $\mathrm{FEV}_{1}<80 \%$ of predicted. Cancer within 5 years prior to inclusion, asthma or lung disorders other than COPD, active inflammatory disease other than COPD, or exacerbations in the last 4 weeks were cause for non-inclusion; patients in the latter category could be re-screened later. The Regional Committee for Medical and Health Research Ethics, region west, approved the study (REK-Vest, case number 165.08). Informed written consent was obtained from all participants.

\section{Data collection}

At inclusion, all patients were examined by a study physician, including a clinical interview regarding respiratory symptoms, smoking history, comorbidities and medication. Comorbidities were pooled to calculate the Charlson Comorbidity Score (CCS) [28]. All patients performed spirometry, before and after bronchodilation with $0.4 \mathrm{mg}$ salbutamol, using a VIASYS MasterScope (VIASYS Healthcare GmbH, Hoechberg, Germany). Patients with COPD were categorised according to the 2007 Global Initiative for Chronic Obstructive Lung Disease (GOLD) guidelines, the current guidelines at the time of inclusion.

Body composition was assessed with bioelectrical impedance measurements. Cachexia was defined as a fat free mass index (FFMI) $<17 \mathrm{~kg} \cdot \mathrm{m}^{-2}$ in men or $<14 \mathrm{~kg} \cdot \mathrm{m}^{-2}$ in women, which corresponds to the lower $95 \%$ confidence limit in a normal population [29]. Obesity was defined as a fat mass index of $>9.3 \mathrm{~kg} \cdot \mathrm{m}^{-2}$ in men or $>13.5 \mathrm{~kg} \cdot \mathrm{m}^{-2}$ in women [29].

The patients were followed for 3 years, with visits and spirometry each 6 months, as well as annual measurement of body composition. The number of exacerbations was assessed via regular phone calls by study personnel, combined with an interview and check of hospital journals at each visit [25]. Only COPD exacerbations treated with either antibiotics, peroral steroids or hospital admission were counted, corresponding to moderate or severe exacerbations [30]. Data on mortality, including cause and date of 
death, were obtained from the Norwegian Cause of Death Registry, ending registration October 2015. In total, 147 patients died within the follow-up period and 17 had an unknown cause of death.

\section{Laboratory measurements}

Peripheral venous blood was drawn into pyrogen-free EDTA collection tubes and centrifuged within $30 \mathrm{~min}$ at $2150 \times \mathrm{g}$ for $15 \mathrm{~min}$ at $4^{\circ} \mathrm{C}$. The plasma was aliquoted and stored in $-80^{\circ} \mathrm{C}$ ultra-freezers until measurements in 2014, meaning that samples were not previously thawed before measurements were taken. Plasma levels of GDF15 were measured in the baseline samples by ELISA (Quantikine, R\&D Systems, Inc., Abingdon, UK). All samples were measured in duplicate, and only accepted if intra-assay variance was $<10 \%$.

\section{Missing data}

18 patients only had spirometry measured at baseline, and 30 patients had only one or no measurements of FFMI. Data from these patients were not included in the longitudinal analysis for each of the two respective outcomes. Further detail is provided in the supplementary material.

\section{Statistical analyses}

The distribution of GDF15 was not normal. GDF15 was evaluated as a continuous variable and as a categorical variable, both dichotomised and in quartiles. Evaluating GDF15 as a dichotomised variable (either high or low levels of GDF15), with a cut-off at the median, gave the best discriminative power for our longitudinal outcomes, as it gave the lowest Akaike Information Criterion value, similarly as seen in other studies [31]. Thus, the analyses presented in this paper are with GDF15 as a categorical variable. Cross-sectional associations between COPD disease characteristics and systemic levels of GDF15 were evaluated by logistic regression, with high-versus-low GDF15 as the outcome variable. The core regression model included sex, age, smoking and comorbidity. Exacerbation count, body composition (both as a dichotomous cachexia variable and with FFMI in four quartiles), FEV1 (both as a continuous variable as per cent predicted and as GOLD category) and FVC (as a continuous variable) were added to the core regression model one at a time owing to potential collinearity. Survival analyses were performed with GDF15 as a predictor variable, adjusting for sex, age, body composition, smoking, GOLD stage, CCS and exacerbation count in the 12 months before inclusion, using Cox regression models estimating hazard ratios (HRs) for both all-cause mortality and mortality due to cancer or cardiovascular or respiratory diseases. The incidence rate ratios (IRRs) of yearly exacerbations were estimated using a negative binomial random effects regression model. Yearly changes in FEV1, FVC and FFMI were analysed using random effects linear regression models. All models adjusted for the same variables as in the survival analysis and Stata 13.1 (StataCorp LP, College Station, TX, USA) was used for the statistical analyses. See the supplementary material for information on missing values.

\section{Results}

The study population is described in table 1 . The median level of GDF15 in 413 patients was $0.86 \mathrm{ng} \cdot \mathrm{mL}^{-1}$ (interquartile range $0.64-1.12 \mathrm{ng} \cdot \mathrm{mL}^{-1}$ ).

\section{Cross-sectional associations between GDF15 and COPD disease characteristics}

At baseline, high levels of GDF15 were associated with increasing age, current smoking and increasing comorbidity, shown in table 2. In addition, added to the same model, high levels of GDF15 were independently associated with $\geqslant 2$ yearly exacerbations before inclusion and with the presence of cachexia (figure 1), especially in the lowest FFMI quartile. Furthermore, GDF15 was associated with low values of FEV1, but not FVC.

\section{GDF15 is a predictor of mortality in COPD}

Patients with high levels of GDF15 at baseline had a HR of 2.1 (95\% CI 1.4-3.1) for all-cause mortality after adjustment for sex, age, body composition, smoking, GOLD stage and CCS (table 3; for all data, see supplementary material, table S1).

Analyses on cause-specific mortality revealed the strongest association between GDF15 and respiratory causes of death. Additional adjustment for heart failure or coronary disease instead of CCS did not significantly change our results (data not shown).

In patients with high GDF15 levels, cachexia was independently associated with higher mortality (HR 2.0, 95\% CI 1.2-3.4), whereas in patients with low GDF15 levels, cachexia did not indicate increased mortality (HR 0.9, 95\% CI 0.4-2.0). However, there was no statistically significant interaction between cachexia and GDF15 levels $(\mathrm{p}=0.17)$ with regards all-cause mortality.

Remarkably, patients with COPD with high levels of GDF15 at baseline continued to have increasingly higher mortality rates for up to 9 years of observation (figure 2). 


\begin{tabular}{|c|c|}
\hline Variables & Subjects $\#$ \\
\hline \multicolumn{2}{|l|}{ Sex } \\
\hline Male & 61.0 \\
\hline Female & 39.0 \\
\hline Age years & $63.4(6.9)$ \\
\hline \multicolumn{2}{|l|}{ Smoking } \\
\hline Ex-smoker & 57.1 \\
\hline Current smoker & 42.9 \\
\hline \multicolumn{2}{|l|}{ Body composition } \\
\hline Normal & 55.5 \\
\hline Obese & 28.3 \\
\hline Cachectic & 16.2 \\
\hline \multicolumn{2}{|l|}{ FEV $1 \%$ predicted } \\
\hline $50-80 \%$ & 47.7 \\
\hline $30-50 \%$ & 41.4 \\
\hline$<30 \%$ & 10.9 \\
\hline \multicolumn{2}{|l|}{ Charlson Comorbidity Score } \\
\hline 1 & 56.9 \\
\hline 2 & 24.2 \\
\hline 3 & 11.6 \\
\hline 4 & 7.3 \\
\hline \multicolumn{2}{|c|}{ Exacerbations 12 months before inclusion } \\
\hline $0-1$ & 83.5 \\
\hline$\geqslant 2$ & 16.5 \\
\hline Plasma level of GDF15 $\mathrm{ng} \cdot \mathrm{mL}^{-1}$ & $0.86(0.64-1.12)$ \\
\hline
\end{tabular}

\section{GDF15 is a predictor of increased COPD exacerbation rate}

We recorded that 353 patients had one or several exacerbations. A total of 1236 exacerbations were counted, of which 880 were considered moderate and 356 severe. High levels of GDF15 were significantly associated with an increase in the IRR for moderate and/or severe COPD exacerbations in the 3 years of follow-up, both in the unadjusted analysis (IRR 1.64, 95\% CI 1.29-2.08) and after adjustment for sex, age, body composition, smoking, GOLD stage and CCS (IRR 1.39, 95\% CI 1.10-1.74) (table 4).

\section{GDF15 is a predictor of increased yearly decline in FEV1 and FVC, but not FFMI}

Patients with high GDF15 levels had an additional yearly decline in predicted FEV1 of $1.04 \%$ and in predicted FVC of $1.19 \%$ compared to patients with low levels of GDF15 ( $p<0.01$ for both) (figure 3). No similar associations were found with yearly decline in FFMI, because levels of baseline GDF15 did not impact the subsequent rate of decline in lean body mass (figure 4). The full analyses of decline in FEV1 and FVC including coefficients for all co-variables are presented in supplementary tables S2 and S3, and for FFMI in supplementary table S4.

\section{Discussion}

Our study showed that plasma levels of GDF15 are associated with several COPD characteristics at baseline, such as smoking, frequent COPD exacerbations before inclusion, cachexia and low levels of FEV1. Further, during follow-up, our study showed that patients with high levels of GDF15 have a higher mortality, an increased COPD exacerbation rate, and a steeper decline in both FEV1 and FVC, all after multivariable adjustment. GDF15 had no predictive value on the development of cachexia, measured as a decline in FFMI.

Our study is in accordance with earlier studies which describe GDF15 as a marker of all-cause mortality [16], as we found a clear correlation between increasing GDF15 and risk of death in patients with COPD, for as long as 9 years of follow-up. The novel findings in our study are that high levels of GDF15 at inclusion predict a higher incidence of COPD exacerbation and a steeper decline in lung function, shown for both FEV1 and FVC. 
TABLE 2 Associations between chronic obstructive pulmonary disease characteristics and high levels of growth differentiation factor-15 using bi- and multivariable logistic regression

\begin{tabular}{|c|c|c|c|c|}
\hline & \multicolumn{2}{|c|}{ Unadjusted } & \multicolumn{2}{|c|}{ Adjusted } \\
\hline & OR $(95 \% \mathrm{CI})$ & p-value & OR $(95 \% \mathrm{CI})$ & p-value \\
\hline \multicolumn{5}{|l|}{ Age } \\
\hline per 10-year increase & $2.48(1.81-3.40)$ & $<0.001$ & $2.73(1.93-3.86)$ & $<0.001$ \\
\hline \multicolumn{5}{|l|}{ Sex } \\
\hline Female & 1 & & 1 & \\
\hline Male & $1.14(0.77-1.70)$ & 0.51 & $0.90(0.59-1.39)$ & 0.64 \\
\hline \multicolumn{5}{|l|}{ Smoking status } \\
\hline Ex-smoker & 1 & & 1 & \\
\hline Current smoker & $1.59(1.08-2.36)$ & 0.02 & $2.48(1 . \overline{5} 8-3.87)$ & $<0.001$ \\
\hline \multicolumn{5}{|c|}{ Charlson Comorbidity Score } \\
\hline 1 & 1 & & 1 & \\
\hline 2 & $1.58(0.99-2.54)$ & 0.06 & $1.43(0.86-2.35)$ & 0.17 \\
\hline 3 & $2.46(1.29-4.69)$ & 0.006 & $1.92(0.97-3.82)$ & 0.06 \\
\hline$\geqslant 4$ & $3.15(1.38-7.17)$ & 0.006 & $2.52(1.05-6.02)$ & 0.04 \\
\hline \multicolumn{5}{|c|}{ Exacerbations 12 months before inclusion ${ }^{\#}$} \\
\hline $0-1$ & 1 & & 1 & \\
\hline$\geqslant 2$ & $2.07(1.21-3.55)$ & 0.008 & $2.28(1.27-4.09)$ & 0.006 \\
\hline \multicolumn{5}{|l|}{ Body composition ${ }^{\#}$} \\
\hline Normal & 1 & & 1 & \\
\hline Cachectic & $2.39(1.51-3.78)$ & $<0.001$ & $1.77(1.07-2.94)$ & 0.03 \\
\hline Obese & $1.30(0.75-2.24)$ & 0.35 & $1.05(0.57-1.92)$ & 0.88 \\
\hline \multicolumn{5}{|l|}{ Free fat mass index $\#$} \\
\hline Q1 (highest) & 1 & & 1 & \\
\hline Q2 & $1.06(0.61-1.83)$ & 0.84 & $1.07(0.59-1.96)$ & 0.82 \\
\hline Q3 & $0.85(0.49-1.47)$ & 0.56 & $0.99(0.49-2.02)$ & 0.99 \\
\hline Q4 (lowest) & $2.85(1.61-5.05)$ & $<0.001$ & $3.25(1.48-7.15)$ & 0.003 \\
\hline FEV1 \% predicted ${ }^{\#, \uparrow}$ & $0.98(0.97-0.99)$ & 0.003 & $0.98(0.97-1.00)$ & 0.01 \\
\hline \multicolumn{5}{|c|}{ GOLD 2007 classification ${ }^{\#}$} \\
\hline FEV $150-80 \%$ & 1 & & 1 & \\
\hline FEV $130-50 \%$ & $1.72(1.14-2.60)$ & 0.01 & $1.58(1.00-2.48)$ & 0.048 \\
\hline FEV $1<30 \%$ & $1.84(0.96-3.55)$ & 0.07 & $2.03(0.99-4.15)$ & 0.053 \\
\hline FVC $\%$ predicted ${ }^{\#, \pi}$ & $0.99(0.98-1.00)$ & 0.07 & $0.99(0.98-1.01)$ & 0.37 \\
\hline
\end{tabular}

FEV1 : forced expiratory volume in $1 \mathrm{~s}$; GOLD: Global Initiative for Chronic Obstructive Lung Disease; FVC: forced vital capacity. " : for multivariable regression, variables added one at a time to the model above; ๆ": per \% increase.

The main strengths of our study were the large number of participants $(n=413)$, combined with longitudinal follow-up for 3 years for all outcomes but mortality, where registration lasted approximately 9 years. The patients underwent comprehensive evaluation both at inclusion and during follow-up, allowing for detailed multivariable regression analysis with adjustment for important co-variables.

FIGURE 1 Growth differentiation factor-15 (GDF15) levels in non-cachectic versus cachectic patients. $p=0.03$ after multivariable adjustment for sex, age, smoking, Global Initiative for Chronic Obstructive Lung Disease stage, Charlson Comorbidity Score and exacerbation count 12 months before inclusion. Outliers are not shown. FFMI: fat free mass index.

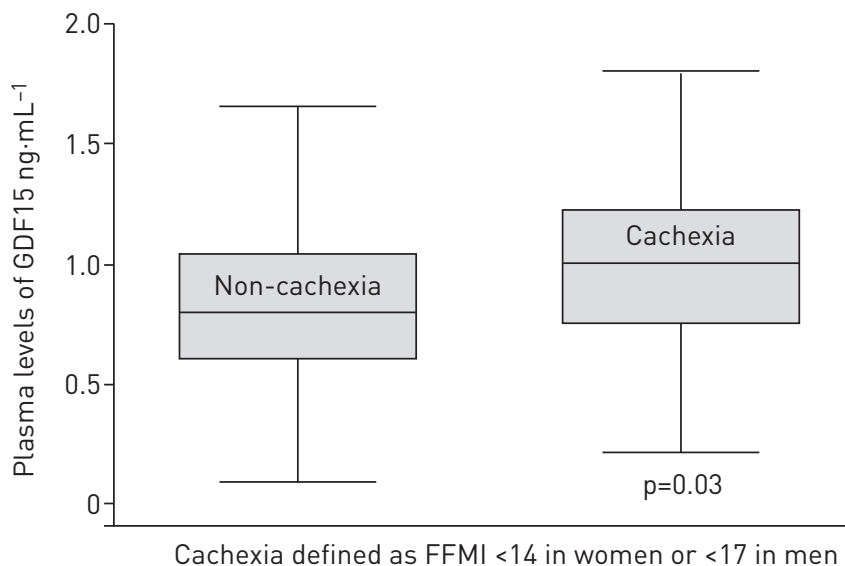

Cachexia defined as FFMI $<14$ in women or $<17$ in men 
TABLE 3 The unadjusted and adjusted hazard ratios for death among patients with chronic obstructive pulmonary disease with high levels of growth differentiation factor-15 at baseline

\begin{tabular}{lcccc} 
Cause of death & Subjects $\mathbf{n}$ & Unadjusted HR (95\% CI) & p-value & Adjusted $^{\#}$ HR (95\% CI) \\
\hline All-cause & 147 & $3.00(2.10-4.27)$ & $<0.001$ & $2.07(1.40-3.05)$ \\
Cancer & 27 & $1.82(0.85-3.94)$ & 0.13 & $1.60(0.70-3.65)$ \\
Cardiovascular & 20 & $3.73(1.36-10.3)$ & 0.01 & $1.72(0.56-5.30)$ \\
Respiratory & 70 & $3.63(2.12-6.21)$ & $<0.001$ & $2.38(1.31-4.31)$ \\
Other/unknown & 30 & $2.76(1.29-5.91)$ & 0.009 & $2.39(1.04-5.49)$ \\
\hline
\end{tabular}

HR: hazard ratio. \#: adjusted for sex, age, body composition, smoking, Global Initiative for Chronic Obstructive Lung Disease stage, Charlson Comorbidity Score and exacerbation count before inclusion.

Some limitations of our study should be mentioned. First, this study is not a randomised controlled study, thus the predictive value of GDF15 cannot be interpreted regarding causality. Second, the selection of patients with COPD was not a random sampling from a general population, but instead a sampling of COPD patients from previous patient studies or outpatient clinics [26]. In addition, GOLD stage 1 patients were not included by design. Our study sample is representative of a population with more advanced COPD, in that half the patients were GOLD stage 3 or 4 . Thus, we would not be able to demonstrate an effect of GDF15 on a decline in FFMI if the effect was exclusively present in early stages of disease. Third, a replication cohort for validation of our findings would strengthen our study. Fourth, there is a lack of longitudinal follow-up for some of the patients, especially among patients with an apparently poor prognosis at inclusion and also with a high mortality, thus our findings in this patient category may be less robust (see supplementary material for details). Finally, although increased systemic levels of GDF15 are associated with both COPD [21] and lung injury [11], GDF15 is not lung specific, and we cannot tell whether the source of GDF15 is from lung tissue or other organ systems.

The mechanisms behind both GDF15 secretion as well as its action on target receptors in the airways are incompletely understood. However, studies by Wu et al. indicated GDF15 induction in airway epithelium after exposure to cigarette smoke $[32,33]$. These authors described activation of both the phosphoinositide 3-kinase and the Smad1 pathways via GDF15, where the activing receptor-like kinase-1 was a possible GDF15 receptor. Wu et al. further found an association between increased airway GDF15 levels and several cellular senescence markers, as well as a potential role for GDF15 in mucin induction. These findings may help explain our findings of increased exacerbation count and a faster lung function decline in patients with high levels of GDF15.

Several studies have linked elevated circulating levels of GDF15 to cardiovascular disease, particularly heart failure [8], but also coronary disease [34] and pulmonary hypertension [35]. These conditions frequently coexist with COPD, and are also associated with adverse outcomes in patients with COPD [36, 37]. Even though high levels of GDF15 have been seen in patients with heart disease, it has been shown that GDF15 is secreted from cardiomyocytes after oxidative stress, and GDF15 has been proposed to have a beneficial anti-inflammatory effect in protecting against myocardial damage [38]; however, the role of GDF15 in cardiovascular disease is not fully understood. Several of our patients with COPD had coexisting cardiovascular disease that could have affected their plasma GDF15 levels. Although adjustment for

FIGURE 2 Survival analysis, high versus low levels of growth differentiation factor-15 (GDF15). $p<0.001$ after multivariable adjustment in a Cox regression model for sex, age, body composition, smoking, Global Initiative for Chronic Obstructive Lung Disease stage, Charlson Comorbidity Score and exacerbation count 12 months before inclusion.

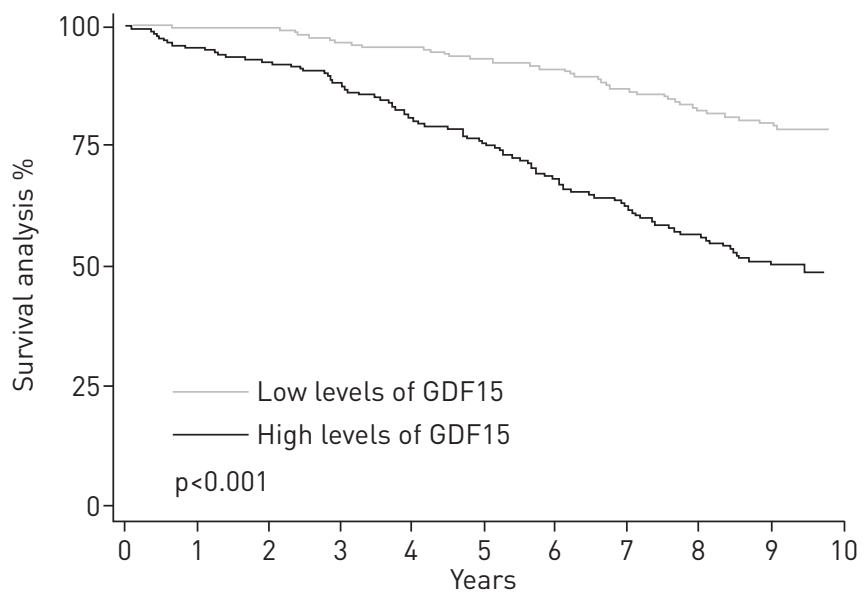


TABLE 4 Multivariable models of the yearly incidence rate ratio of moderate or severe chronic obstructive pulmonary disease exacerbations, estimated by a random effects negative binomial model

\begin{tabular}{|c|c|c|}
\hline Baseline explanatory variables & $\operatorname{IRR}(95 \% \mathrm{CI})$ & p-value \\
\hline \multicolumn{3}{|l|}{ Sex } \\
\hline Men & 1 & \\
\hline Women & $1.35(1.08-1.69)$ & 0.01 \\
\hline \multicolumn{3}{|l|}{ Age } \\
\hline per 10-year increase & $1.10(0.92-1.32)$ & 0.28 \\
\hline \multicolumn{3}{|l|}{ Body composition } \\
\hline Normal & 1 & \\
\hline Cachectic & $1.01(0.77-1.31)$ & 0.96 \\
\hline Obese & $1.18(0.87-1.61)$ & 0.28 \\
\hline \multicolumn{3}{|l|}{ Smoking } \\
\hline Ex-smoker & 1 & \\
\hline Current smoker & $0.98(0.77-1.24)$ & 0.84 \\
\hline \multicolumn{3}{|c|}{ Exacerbations 12 months before inclusion } \\
\hline $0-1$ & 1 & \\
\hline$\geqslant 2$ & $2.03(1.55-2.66)$ & $<0.001$ \\
\hline \multicolumn{3}{|l|}{ GOLD 2010 classification } \\
\hline FEV $150-80 \%$ & 1 & \\
\hline FEV $130-50 \%$ & $1.59(1.26-2.01)$ & $<0.001$ \\
\hline FEV $1<30 \%$ & $3.09(2.15-4.43)$ & $<0.001$ \\
\hline \multicolumn{3}{|l|}{ Charlson Comorbidity Score } \\
\hline 1 & 1 & \\
\hline 2 & $0.94(0.72-1.23)$ & 0.66 \\
\hline 3 & $1.00(0.70-1.44)$ & 0.98 \\
\hline$\geqslant 4$ & $1.07(0.69-1.66)$ & 0.77 \\
\hline \multicolumn{3}{|l|}{ GDF15 } \\
\hline Low levels" & 1 & \\
\hline High levels & $1.39(1.10-1.74)$ & 0.006 \\
\hline
\end{tabular}

IRR: incidence rate ratio; GOLD: Global Initiative for Chronic Obstructive Lung Disease; FEV1: forced expiratory volume in $1 \mathrm{~s}$; GDF15: growth differentiation factor-15. * : cut-off median.

comorbidities including known cardiovascular disease did not change our results, we cannot exclude the impact from these conditions on either measured GDF15 levels or on adverse outcomes in our patients.

Cachexia is another condition frequently seen in COPD. It is associated with increased disease burden and mortality but its causes are multifactorial and it remains difficult to treat [39]. Several studies have described cachexia as being closely related to elevated levels of GDF15, especially in cachexia related to cancer [18-20], and cachexia has also recently been described in COPD [22]. It has been suggested that GDF15 has a suppressive effect on body weight both via appetite control on the hypothalamus and via peripheral regulation of energy metabolism $[18,40]$. In our study, high levels of GDF15 were a strong independent predictor for increased mortality regardless of the patient's nutritional status. Interestingly, cachexia was not associated with increased mortality in patients with low GDF15 levels, indicating an upstream role for GDF15 in cachexia development. However, although we found a clear relationship between GDF15 and cachexia at inclusion, having high levels of GDF15 did not predict increased decline of FFMI in the longitudinal analysis.

The search for biomarkers in COPD is important for several reasons. There exists no useful biomarker for the diagnosis of COPD, or for a COPD exacerbation. Further, it is important to identify patients with increased risk for adverse outcomes in order to intensify observation and treatment in these patients. Although GDF15 lacks the combined sensitivity and specificity of an ideal biomarker in COPD, it could give prognostic information in addition to other easily accessible clinical parameters. Finally, the investigation of GDF15 and other biomarkers could provide valuable insight into the pathophysiology behind the disease, and the markers could themselves be targets for pharmacologic treatment. In murine models, treatment with antibodies targeting GDF15 has shown reversal of cancer-related cachexia after only a single injection $[17,41]$. The association between elevated GDF15 and several adverse outcomes in patients with COPD makes GDF15 a potential treatment target of high interest. 
FIGURE 3 Modelled decline in forced expiratory volume in $1 \mathrm{~s}$ (FEV 1 ) and forced vital capacity (FVC) in patients with high versus low levels of growth differentiation factor-15 (GDF15) using a random effects linear regression model. $p<0.01$ after multivariable adjustment for sex, age, body composition, smoking, Global Initiative for Chronic Obstructive Lung Disease stage, Charlson Comorbidity Score and exacerbation count 12 months before inclusion.

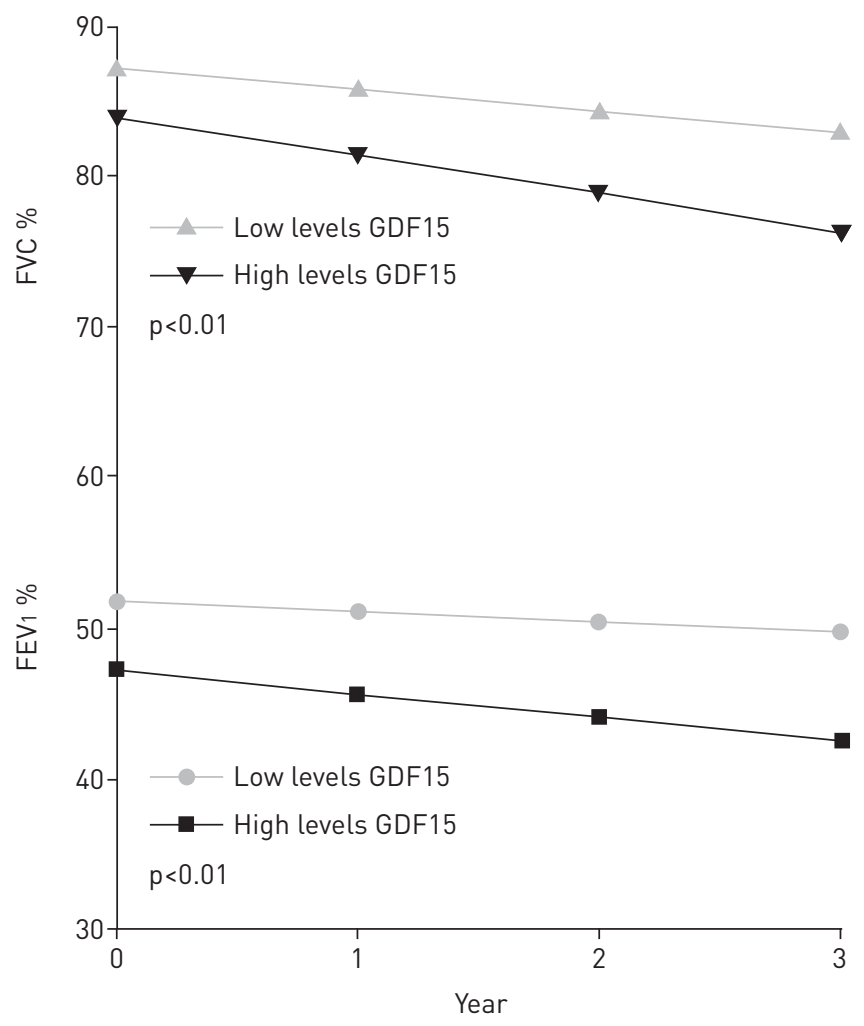

Although its biological mechanisms remain incompletely understood, GDF15 emerges as a central biomarker in a variety of conditions. We show that elevated GDF15 levels can predict several adverse outcomes in COPD. To our knowledge, these have not been described in earlier studies. The existence of inhibitory antibodies and their therapeutic potential makes GDF15 even more intriguing. Nevertheless,

FIGURE 4 Modelled decline in fat free mass index (FFMI) in patients with high versus low levels of growth differentiation factor-15 [GDF15] using a random effects linear regression model. $p=0.77$ after multivariable adjustment for sex, age, body composition, smoking, Global Initiative for Chronic Obstructive Lung Disease stage, Charlson Comorbidity Score and exacerbation count 12 months before inclusion.

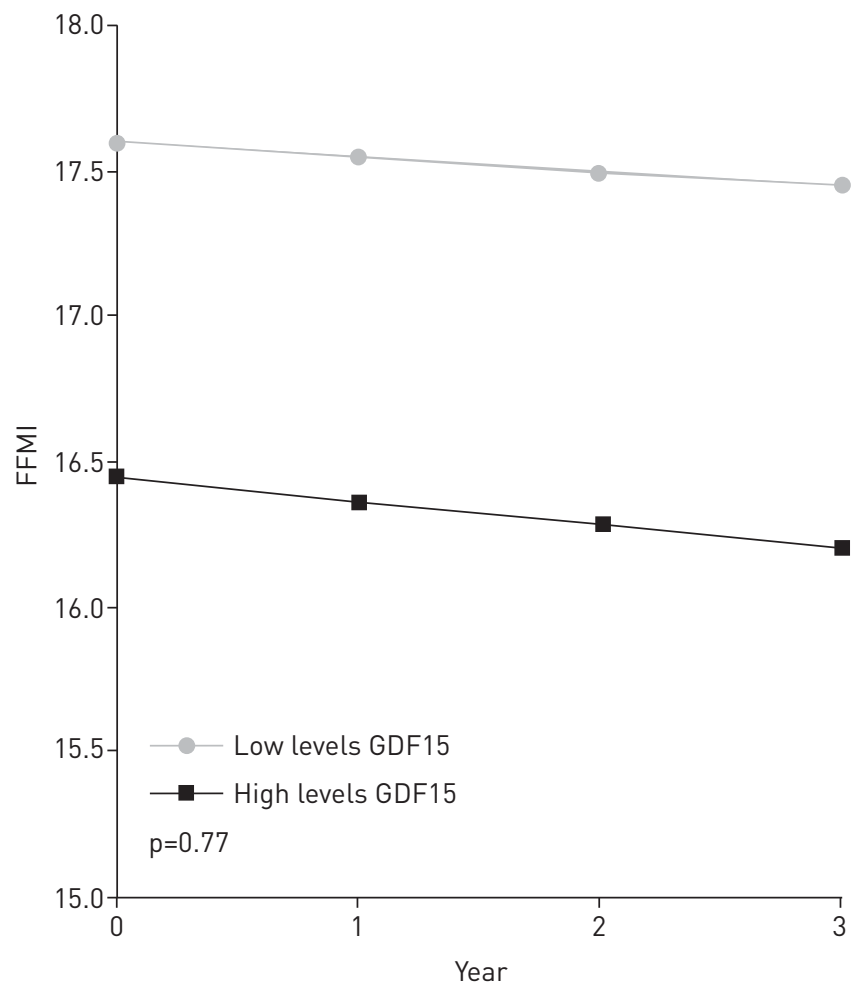


more research on GDF15 and its biochemical properties is needed to understand its role in COPD especially, as well as in general pathophysiology.

\section{Acknowledgements}

Author contributions: G.R. Husebø, J.A. Hardie, P.S. Bakke and T.M. Eagan designed the study. G.R. Husebø,

R. Grønseth, J.A. Hardie, P.S. Bakke, L. Lerner, J. Gyuris and T.M. Eagan obtained the data. G.R. Husebø and T.M. Eagan analysed the data and drafted the manuscript. All authors have seen and approved the final version of the manuscript.

\section{References}

$1 \quad$ López-Campos JL, Tan W, Soriano JB. Global burden of COPD. Respirology 2016; 21: 14-23.

2 Agusti A, Sobradillo P, Celli B. Addressing the complexity of chronic obstructive pulmonary disease: from phenotypes and biomarkers to scale-free networks, systems biology, and P4 medicine. Am J Respir Crit Care Med 2011; 183: 1129-1137.

3 GOLD. The Global Initiative for Chronic Obstructive Lung Disease (GOLD). 2015 Date last accessed and updated: June 1, 2016.

4 Böttner M, Suter-Crazzolara C, Schober A, et al. Expression of a novel member of the TGF-beta superfamily, growth/differentiation factor-15/macrophage-inhibiting cytokine-1 (GDF-15/MIC-1) in adult rat tissues. Cell Tissue Res 1999; 297: 103-110.

5 Bootcov MR, Bauskin AR, Valenzuela SM, et al. MIC-1, a novel macrophage inhibitory cytokine, is a divergent member of the TGF-beta superfamily. Proc Natl Acad Sci USA 1997; 94: 11514-11519.

6 Breit SN, Johnen H, Cook AD, et al. The TGF-beta superfamily cytokine, MIC-1/GDF15: a pleotrophic cytokine with roles in inflammation, cancer and metabolism. Growth Factors 2011; 29: 187-195.

7 Fairlie WD, Moore AG, Bauskin AR, et al. MIC-1 is a novel TGF-beta superfamily cytokine associated with macrophage activation. J Leukoc Biol 1999; 65: 2-5.

8 Wollert KC, Kempf T. Growth differentiation factor 15 in heart failure: an update. Curr Heart Fail Rep 2012; 9: 337-345.

9 Berezin AE. Diabetes mellitus related biomarker: the predictive role of growth-differentiation factor-15. Diabetes Metab Syndr 2016; 10: 1 Suppl. 1, S154-S157.

10 Guenancia C, Kahli A, Laurent G, et al. Pre-operative growth differentiation factor 15 as a novel biomarker of acute kidney injury after cardiac bypass surgery. Int J Cardiol 2015; 197: 66-71.

11 Zimmers TA, Jin X, Hsiao EC, et al. Growth differentiation factor-15/macrophage inhibitory cytokine-1 induction after kidney and lung injury. Shock 2005; 23: 543-548.

12 Staff AC, Bock AJ, Becker C, et al. Growth differentiation factor-15 as a prognostic biomarker in ovarian cancer. Gynecol Oncol 2010; 118: 237-243.

13 Wallin U, Glimelius B, Jirstrom K, et al. Growth differentiation factor 15: a prognostic marker for recurrence in colorectal cancer. Br J Cancer 2011; 104: 1619-1627.

14 Baek KE, Yoon SR, Kim JT, et al. Upregulation and secretion of macrophage inhibitory cytokine-1 (MIC-1) in gastric cancers. Clin Chim Acta 2009; 401: 128-133.

15 Corre J, Hebraud B, Bourin P. Concise review: growth differentiation factor 15 in pathology: a clinical role? Stem Cells Transl Med 2013; 2: 946-952.

16 Wiklund FE, Bennet AM, Magnusson PK, et al. Macrophage inhibitory cytokine-1 (MIC-1/GDF15): a new marker of all-cause mortality. Aging Cell 2010; 9: 1057-1064.

17 Johnen $\mathrm{H}$, Lin S, Kuffner T, et al. Tumor-induced anorexia and weight loss are mediated by the TGF-beta superfamily cytokine MIC-1. Nat Med 2007; 13: 1333-1340.

18 Tsai VW, Husaini Y, Manandhar R, et al. Anorexia/cachexia of chronic diseases: a role for the TGF-beta family cytokine MIC-1/GDF15. J Cachexia Sarcopenia Muscle 2012; 3: 239-243.

19 Lerner L, Hayes TG, Tao N, et al. Plasma growth differentiation factor 15 is associated with weight loss and mortality in cancer patients. J Cachexia Sarcopenia Muscle 2015; 6: 317-324.

20 Lerner L, Tao J, Liu Q, et al. MAP3K11/GDF15 axis is a critical driver of cancer cachexia. J Cachexia Sarcopenia Muscle 2015; 7: 467-482.

21 Mutlu LC, Altintas N, Aydin M, et al. Growth differentiation factor-15 is a novel biomarker predicting acute exacerbation of chronic obstructive pulmonary disease. Inflammation 2015; 38: 1805-1813.

22 Patel MS, Lee J, Baz M, et al. Growth differentiation factor-15 is associated with muscle mass in chronic obstructive pulmonary disease and promotes muscle wasting in vivo. J Cachexia Sarcopenia Muscle 2015; 7: 436-448.

23 Freeman $\mathrm{CM}$, Martinez $\mathrm{CH}$, Todt JC, et al. Acute exacerbations of chronic obstructive pulmonary disease are associated with decreased CD4+ \& CD8+ T cells and increased growth \& differentiation factor-15 (GDF-15) in peripheral blood. Respir Res 2015; 16: 94.

24 Kim M, Cha SI, Choi KJ, et al. Prognostic value of serum growth differentiation factor-15 in patients with chronic obstructive pulmonary disease exacerbation. Tuberc Respir Dis 2014; 77: 243-250.

25 Husebø GR, Bakke PS, Aanerud M, et al. Predictors of exacerbations in chronic obstructive pulmonary disease--results from the Bergen COPD cohort study. PloS One 2014; 9: e109721.

26 Eagan TM, Ueland T, Wagner PD, et al. Systemic inflammatory markers in COPD: results from the Bergen COPD Cohort Study. Eur Respir J 2010; 35: 540-548.

27 Eagan TM, Gabazza EC, D'Alessandro-Gabazza C, et al. TNF-alpha is associated with loss of lean body mass only in already cachectic COPD patients. Respir Res 2012; 13: 48.

28 Charlson ME, Pompei P, Ales KL, et al. A new method of classifying prognostic comorbidity in longitudinal studies: development and validation. J Chronic Dis 1987; 40: 373-383.

29 Schutz Y, Kyle UU, Pichard C. Fat-free mass index and fat mass index percentiles in Caucasians aged 18-98 y. Int J Obes Relat Metab Disord 2002; 26: 953-960.

30 Rodriguez-Roisin R. Toward a consensus definition for COPD exacerbations. Chest 2000; 117: 5 Suppl. 2, 398S-401S. 
31 Kempf T, von Haehling S, Peter T, et al. Prognostic utility of growth differentiation factor-15 in patients with chronic heart failure. J Am Coll Cardiol 2007; 50: 1054-1060.

$32 \mathrm{Wu} \mathrm{Q}$, Jiang $\mathrm{D}$, Chu HW. Cigarette smoke induces growth differentiation factor 15 production in human lung epithelial cells: implication in mucin over-expression. Innate Immun 2012; 18: 617-626.

$33 \mathrm{Wu} \mathrm{Q}$, Jiang $\mathrm{D}$, Matsuda JL, et al. Cigarette smoke induces human airway epithelial senescence via growth differentiation factor 15 production. Am J Respir Cell Mol Biol 2016; 55: 429-438.

34 Wollert KC, Kempf T, Peter T, et al. Prognostic value of growth-differentiation factor-15 in patients with non-ST-elevation acute coronary syndrome. Circulation 2007; 115: 962-971.

35 Nickel $\mathrm{N}$, Kempf $\mathrm{T}$, Tapken $\mathrm{H}$, et al. Growth differentiation factor-15 in idiopathic pulmonary arterial hypertension. Am J Respir Crit Care Med 2008; 178: 534-541.

36 Wells JM, Washko GR, Han MK, et al. Pulmonary arterial enlargement and acute exacerbations of COPD. $N$ Engl J Med 2012; 367: 913-921.

37 Mascarenhas J, Azevedo A, Bettencourt P. Coexisting chronic obstructive pulmonary disease and heart failure: implications for treatment, course and mortality. Curr Opin Pulm Med 2010; 16: 106-111.

38 Kempf T, Zarbock A, Widera C, et al. GDF-15 is an inhibitor of leukocyte integrin activation required for survival after myocardial infarction in mice. Nat Med 2011; 17: 581-588.

39 Sanders KJ, Kneppers AE, van de Bool C, et al. Cachexia in chronic obstructive pulmonary disease: new insights and therapeutic perspective. J Cachexia Sarcopenia Muscle 2016; 7: 5-22.

40 Tsai VW, Macia L, Johnen H, et al. TGF-b superfamily cytokine MIC-1/GDF15 is a physiological appetite and body weight regulator. PloS One 2013; 8: e55174.

41 Lerner L, Tao N, Krieger B, et al. Abstract 1153: Effective treatment of cancer associated cachexia by AV-380, a GDF15 inhibitory antibody. Cancer Res 2015; 75: 15 Suppl., 1153. 